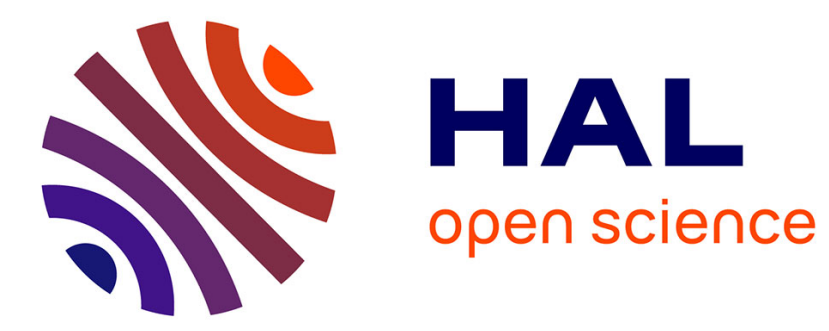

\title{
A New Method To Design Balanced Space-Time Trellis Codes for Several Transmit Antennas
}

Pierre Viland, Gheorghe I. Zaharia, Jean-François Hélard

\section{To cite this version:}

Pierre Viland, Gheorghe I. Zaharia, Jean-François Hélard. A New Method To Design Balanced SpaceTime Trellis Codes for Several Transmit Antennas. GLOBECOM 2008, Nov 2008, New orleans, United States. pp. 1-5, 10.1109/GLOCOM.2008.ECP.234 . hal-00348789

\section{HAL Id: hal-00348789 \\ https://hal.science/hal-00348789}

Submitted on 22 Dec 2008

HAL is a multi-disciplinary open access archive for the deposit and dissemination of scientific research documents, whether they are published or not. The documents may come from teaching and research institutions in France or abroad, or from public or private research centers.
L'archive ouverte pluridisciplinaire HAL, est destinée au dépôt et à la diffusion de documents scientifiques de niveau recherche, publiés ou non, émanant des établissements d'enseignement et de recherche français ou étrangers, des laboratoires publics ou privés. 


\title{
A New Method To Design Balanced Space-Time Trellis Codes for Several Transmit Antennas
}

\author{
Pierre Viland Gheorghe Zaharia Jean-François Hélard \\ Institute for Electronics and Telecommunications of Rennes (IETR) - UMR CNRS 6164 \\ INSA - 20 avenue des Buttes de Coësmes, \\ 35043 Rennes, France \\ Email: pierre.viland@ens.insa-rennes.fr
}

\begin{abstract}
Recently, a new class of trellis codes called "balanced" space-time trellis codes has been proposed. These codes are "balanced" because they use the points of the MIMO constellation with the same probability. In this paper, we propose a new and simple method to design these codes for 4-PSK and 8PSK modulations and several transmit antennas. This design method can be easily extended to $2^{n}$-PSK modulations. Furthermore, new balanced STTC with 3 and 4 transmit antennas are given with better performance than the previously published codes.
\end{abstract}

Keywords - space-time trellis codes, balanced codes, MIMO systems, design method.

\section{INTRODUCTION}

Space time trellis coded modulation (STTCM) was introduced in 1998 by Tarokh et al. [1] by combining channel coding with the multiple input multiple output (MIMO) concept to improve the data rate and the reliability of wireless communications. Several performance criteria have been established to maximize both diversity and coding gain of STTC. The rank and determinant criteria for slow fading channels with the Euclidian distance and the product distance criterion for fast fading channels have been proposed in [1]. In [2] Chen introduced the trace criterion which governs the coding for systems with large transmit or/and receive diversity.

Based on above criteria, many different STTC for 2 Tx antennas have been found by a systematic code search [3-6]. The performance study of these codes was carried out over slow and fast Rayleigh fading channels to identify the most efficient ones. It has been shown that over slow fading channels, the codes constructed with the trace criterion give similar or even better results that the codes constructed with the rank and the determinant criteria. Over fast fading channels, "trace criterion codes" as Chen's codes outperform the other tested codes. In the same way, some codes for $3 \mathrm{Tx}$ antennas have been published in [5], [7]. All the codes which achieve the best performance have the same property: they use the points of the MIMO constellation with the same probability if the data are generated by a binary memoryless source with equally probable symbols. Therefore, these codes where called "Balanced-STTC" (B-STTC) [8]. Thus, the search of good STTC can be reduced to this class. A first construction method to design B-STTC has been proposed in [8]. In this paper, we present a second method to design this class of codes which is simpler than the previous one. Besides, we propose new balanced codes with better performance than the previously published STTC.

The rest of the paper is organised as follows: section II presents briefly the space-time trellis coding. In section III, the performance criteria of STTC are reminded. In section IV, the properties of balanced codes are introduced. In section $\mathrm{V}$, the new method to design B-STTC is presented for 4-PSK and 8-PSK and several transmit antennas. Finally, the paper proposes new balanced codes which outperform the best known STTC.

\section{SPACE TIME TRELlis CODING}

We consider the case of $2^{n}$-PSK ST trellis encoder [8]. This encoder has one input block of $n$ bits and $v$ memory blocks of $\mathrm{n}$ bits. At each time $t \in \mathbb{Z}$, the bits of a block are replaced by the $n$ bits of the previous block. The $i^{t h}$ bit $x_{i}^{t-j+1}, i=1 \ldots n$, of the $j^{\text {th }}$ block, $j=1 \ldots(v+1)$, is associated to $n_{T}$ multiplier coefficients $g_{i, j}^{k} \in \mathbb{Z}_{2^{n}}, k=1 \ldots n_{T}$, where $n_{T}$ is the number of transmit antennas. A ST trellis encoder is thus usually defined by its generator matrix $\boldsymbol{G}$ of $n_{T} \times n(v+1)$ coefficients:

$$
\boldsymbol{G}=\left[\begin{array}{ccccccc}
g_{1,1}^{1} & \ldots & g_{n, 1}^{1} & \ldots & g_{1, v+1}^{1} & \ldots & g_{n, v+1}^{1} \\
\vdots & & \ldots & & \ldots & & \vdots \\
g_{1,1}^{k} & \ldots & g_{n, 1}^{k} & \ldots & g_{1, v+1}^{k} & \ldots & g_{n, v+1}^{k} \\
\vdots & & \ldots & & \ldots & & \vdots \\
g_{1,1}^{n_{T}} & \ldots & g_{n, 1}^{n_{T}} & \ldots & g_{1, v+1}^{n_{T}} & \ldots & g_{n, v+1}^{n_{T}}
\end{array}\right]
$$

The encoder outputs for the $k^{\text {th }}$ antenna are computed as

$$
y_{t}^{k}=\sum_{i=1}^{n} \sum_{j=1}^{v+1} x_{i}^{t-j+1} g_{i, j}^{k} \quad \bmod 2^{n}
$$

and sent to the $k^{\text {th }}$ antenna. The modulated streams for all antennas are then transmitted simultaneously.

\section{DESIGN CRITERIA}

Design criteria have been proposed in [1-2] to exploit the spatial diversity and to offer optimal coding gain. Cases of 
slow and fast Rayleigh fading channels are mainly studied. The transmitted $n_{T}$ dimension symbols $s_{t}=\left[s_{t}^{1} s_{t+1}^{2} \ldots s_{t}^{n_{T}}\right]^{T}$, where $[\cdot]^{T}$ denotes the transpose operator, are assumed to be grouped in a frame of length $L_{f}$. For each case, design criteria are derived from the minimization of the pairwise error probability (PEP), i.e. the probability of transmitting the $n_{T} \times L_{f}$ dimension coded frame $S=\left[s_{t} s_{t+1} \ldots s_{t+L_{f}-1}\right]$ and deciding erroneously in favour of another $n_{T} \times L_{f}$ dimension coded frame $\boldsymbol{E}=\left[e_{t} e_{t+1} \ldots e_{t+L_{f}-1}\right]$. The $n_{T} \times n_{T}$ product matrix $\boldsymbol{A}=$ $\boldsymbol{B} . \boldsymbol{B}^{*}$ is introduced, where $\boldsymbol{B}^{*}$ denotes the hermitian of the $n_{T}$ $\times L_{f}$ difference matrix $\boldsymbol{B}=\boldsymbol{E}-\boldsymbol{S}$.

$$
\boldsymbol{B}=\left[\begin{array}{ccccc}
e_{t}^{1}-s_{t}^{1} & \ldots & e_{t+q}^{1}-s_{t+q}^{1} & \ldots & e_{t+L_{f}-1}^{1}-s_{t+L_{f}-1}^{1} \\
\ldots & \ldots & \ldots & \ldots & \ldots \\
e_{t}^{n_{T}}-s_{t}^{n_{T}} & \ldots & e_{t+q}^{n_{T}}-s_{t+q}^{n_{T}} & \ldots & e_{t+L_{f}-1}^{n_{T}}-s_{t+L_{f}-1}^{n_{T}}
\end{array}\right]
$$

For slow Rayleigh fading, if $\operatorname{rank}(\boldsymbol{A}) \cdot n_{R} \leq 3$, criteria based on rank and determinant (called RDC) are proposed. To obtain good performance, the matrices $\boldsymbol{A}$ have to be full rank matrices and $\min (\operatorname{det}(\boldsymbol{A}))$ computed for all couples of coded frames $(\boldsymbol{S}, \boldsymbol{E})$ must be maximum [1]. Besides, the coding gain is given by $\eta=\sum_{d} N(d) d^{-n_{R}}$, where $N(d)$ is defined as the average number of error events with determinant $d$. The best code must have the minimum value of $\eta$ [9].

In [2], Chen proposes a new criterion to be used in the case of slow and fast Rayleigh fading channels if $\operatorname{rank}(\boldsymbol{A}) \cdot n_{R}>3$. Under this assumption, the PEP is minimized if the sum of all the eigenvalues of the product matrix is maximized. For a square matrix, the sum of all the eigenvalues is equal to the trace of the matrix $\boldsymbol{A}$.

$$
\operatorname{tr}(\boldsymbol{A})=\sum_{k=1}^{n_{T}} \lambda_{k}=\sum_{k=1}^{n_{T}}\left(\sum_{q=0}^{L_{f}-1}\left|e_{t+q}^{k}-s_{t+q}^{k}\right|^{2}\right)
$$

For each pair of coded frames, the matrix $\boldsymbol{A}$ and then $\operatorname{tr}(\boldsymbol{A})$ can be computed. The minimum trace is the minimum of all these values $\operatorname{tr}(\boldsymbol{A})$. When $\operatorname{rank}(\boldsymbol{A}) \cdot n_{R}>3$, the minimization of the PEP amounts to use a code which has the maximum value of the minimum trace. In [9], it is also stated that to minimize the frame error rate (FER), the number of error events with minimum trace has to be minimized. This criterion is called euclidian distance criterion (EDC).

\section{BALANCED CODES}

\section{A. What is a "balanced code"?}

This concept of "balanced codes" has been proposed in [8]. It is based on the observation that the best STTC proposed in the literature present the same property: the generated symbols of the MIMO constellation are equally probable.

If the binary input data are generated by a memoryless binary source $S=\{0,1\}$ with equally probable symbols, then, in the case of $2^{n}$-PSK modulation, from a given state
$\boldsymbol{X}=\left[x_{1} x_{2} \ldots x_{L}\right]^{T} \in \mathbb{Z}_{2}^{L}$ of the $L=n(v+1)$ length shift register realized by $(\mathrm{v}+1)$ blocks of $\mathrm{n}$ bits, the MIMO symbols $\boldsymbol{Y}=\left[y_{1} y_{2} \ldots y_{n_{T}}\right]^{T} \in \mathbb{Z}_{2^{n}}^{n_{T}}$ generated by the STTC encoder is

$$
\boldsymbol{Y}=\boldsymbol{G} . \boldsymbol{X}
$$

where $\boldsymbol{G}$ is the generator matrix (1). This is a deterministic relation. Therefore, the STTC is defined by a map :

$$
\Phi: \mathbb{Z}_{2}^{L} \rightarrow \mathbb{Z}_{2^{n}}^{n_{T}}
$$

which associates to the state $\boldsymbol{X}$ a unique codeword $\boldsymbol{Y}$. Note that $\Phi\left(\mathbb{Z}_{2}^{L}\right) \subseteq \mathbb{Z}_{2^{n}}^{n_{T}}$ represents the set of generated codewords $\boldsymbol{Y}$.

By definition, a STTC is balanced if and only if each generated codeword $\boldsymbol{Y} \in \Phi\left(\mathbb{Z}_{2}^{L}\right)$ has the same number of occurrences:

$$
n(\boldsymbol{Y})=n_{0} \in \mathbb{N}^{*}, \forall \boldsymbol{Y} \in \mathbb{Z}_{2^{n}}^{n_{T}}
$$

For balanced codes, the number of generated codewords is $\operatorname{card}\left(\Phi\left(\mathbb{Z}_{2}^{L}\right)\right)=\frac{\operatorname{card}\left(\Phi\left(\mathbb{Z}_{2^{n}}^{n_{T}}\right)\right)}{2^{n_{0}-1}}$. If $n_{0}=1$, the code is called fully balanced. In this case $\Phi\left(\mathbb{Z}_{2}^{L}\right)=\mathbb{Z}_{2^{n}}^{n_{T}}$ i.e:

$$
\left\{\sum_{j=1}^{j=L}\left(x_{j} \cdot G_{j}\right) \bmod 2^{n} / x_{j} \in\{0,1\}\right\}=\mathbb{Z}_{2^{n}}^{n_{T}}
$$

where $G_{j}$ is the $\mathrm{j}^{\text {th }}$ column of the matrix $\boldsymbol{G}$.

Due to the random memoryless source $\mathbf{S}=\{0,1\}$ with $p(0)=p(1)=1 / 2$, for a given state $\mathbf{X}$, the shift-register of the encoder has only $2^{n}$ equally probable next states. The matrix $\mathbf{T}$ of the transition probabilities between these states corresponds to a Markov chain. Due to the symmetry of the matrix $\mathbf{T}$, the steady-state probabilities of the states $\mathbf{X}$ are all equal. For a balanced code, by using (5), the generated codewords $\boldsymbol{Y}$ are also equally probable. In other words, the generated symbols of the MIMO constellation are equally probable.

\section{B. General properties of balanced codes}

Property 1: If a MIMO code with a L-length shift register is fully balanced then $L \geq L_{\text {min }}=\operatorname{dim}\left(\mathbb{Z}_{2^{n}}^{n_{T}}\right)=n . n_{T}$.

Property 2: Les us consider a fully balanced MIMO with a $L$-length shift-register. Then, for any additional column matrix $G_{i} \in \mathbb{Z}_{2^{n}}^{n_{T}}$, the resulting matrix $\boldsymbol{G}^{\prime}=\left[\boldsymbol{G} G_{i}\right]$ code corresponds to a new balanced code with a $(L+1)$-length shift-register.

Property 3: Each permutation of the rows/columns of the generator matrix $\boldsymbol{G}$ of a B-STTC gives the generator matrix of another B-STTC.

Property 4: A code with $L_{m i n}$-length shift register is fully balanced if and only if the set of columns of the generator matrix $\boldsymbol{G}$ is a base of $\mathbb{Z}_{2^{n}}^{n_{T}}$.

Property 5: $C_{[0]}=2^{n-1} \mathbb{Z}_{2}^{n_{T}}$ is a subgroup of $\mathbb{Z}_{2^{n}}^{n_{T}}$ such as $v=-v, \forall v \in C_{[0]}$. In the case of 4-PSK modulation, each 
element $v \in C_{[0]}$ can be written as $v=2 p$, with $p \in \mathbb{Z}_{2}^{n_{T}}$. The coset $C_{[p]}=p+C_{[0]}$ is called "relative to $v$ ". So, it is possible to write : $\mathbb{Z}_{4}^{n_{T}}=C_{[0]} \cup E_{1}$ with $E_{1}$ the set of the cosets $C_{[p]} \neq C_{[0]}$. In the case of 8-PSK modulation, each element $v \in C_{[0]}=4 \mathbb{Z}_{2}^{n_{T}}$ can be written as $v=2 p$, with $p \in 2 \mathbb{Z}_{2}^{n_{T}}$. For 8-PSK, we note by $E_{1}$ the set of cosets which can be written as $C_{[p]}=p+C_{[0]} \quad$ with $\quad p \in 2 \mathbb{Z}_{2}^{n_{T}} \backslash\left\{\left[\begin{array}{ll}0 & 0\end{array}\right]^{T}\right\}$. So, each coset $C_{[p]}=p+C_{[0]} \in E_{1} \quad$ is called "relative to $v$ ", with $v=2 p \in C_{[0]}$. Each element $q \in E_{1}$ can be written as $q=2 r$, with $r \in \mathbb{Z}_{4}^{n_{T}} \backslash 2 \mathbb{Z}_{2}^{n_{T}}$ and a new set $E_{2}$ of cosets $C_{[q]}=r+C_{[0]}$ can be created. Thus, each coset of $E_{2}$ is also " relative to a element of $E_{l}$, . Therefore, it is possible to write: $\mathbb{Z}_{8}^{n_{T}}=C_{[0]} \cup E_{1} \cup E_{2}$.

\section{A General MeThod to DESIGN 4-PSK AND 8-PSK BALANCED STTC WITH $\mathrm{n}_{\mathrm{T}}$ ANTENNAS}

\section{A. Design of fully balanced codes for 4-PSK and 8-PSK}

Due to Property 2 of the previous section, it is enough to design fully balanced codes with $\mathrm{L}=\mathrm{L}_{\text {min }}$. This new design method must respect the rules:

1. Choose a first no null column $G_{1}=2 p_{1} \in C_{[0]}$. Due to property $4, G_{1}=-G_{1}$, so $H_{1}=\left\{0, G_{1}\right\}$ is a subgroup of $\mathbb{Z}_{2^{n}}^{n_{T}}$ with $n=2$ for 4 -PSK modulation and $n=3$ for 8 -PSK modulation

2. Each new chosen column of $\boldsymbol{G}$ must create a new subgroup $H_{i+1}$ of $\mathbb{Z}_{2^{n}}^{n_{T}}$ with $H_{i} \subset H_{i+1}$ and $\operatorname{card}\left(H_{i+1}\right)=2 \operatorname{card}\left(H_{i}\right)$.

Thus, in order to obtain a fully balanced STTC, the columns of $\boldsymbol{G}$ can be selected as follows:

- $\quad$ if $i<L_{\min }$ columns of $\boldsymbol{G}$ have been already chosen in $\mathbb{Z}_{2^{n}}^{n_{T}}$ and $\left\{\sum_{j=1}^{i} x_{j} G_{j} \bmod 2^{n} / x_{j} \in\{0,1\}\right\}=H_{i}$ is a subgroup of $\mathbb{Z}_{2^{n}}^{n_{T}}$, then the column $G_{i+1}$ of $\boldsymbol{G}$ must belong to $\mathbb{Z}_{2^{n}}^{n_{T}} \backslash H_{i}$ and must be selected in the cosets relative to the vectors which belong to $H_{i}$ or in the coset $C_{[0]}$. This method ensures that $H_{i+1}=H_{i} \cup\left(H_{i}+G_{i+1}\right)$ is a subgroup of $\mathbb{Z}_{2^{n}}^{n_{T}}$. The algorithm ends when $\mathrm{i}=\mathrm{L}_{\text {min }}$.

In order to obtain balanced codes (not necessary fully balanced) the algorithm can be ended at $\mathrm{i}_{0} \leq \mathrm{L}_{\min }-2$. The obtained code is balanced but not fully balanced. One can add a new column $G_{i_{0}+1} \in \mathbb{Z}_{2^{n}}^{n_{T}} \backslash H_{i_{0}}$. Once again, the obtained code is balanced but not fully balanced. If necessary, several elements of $H_{i_{0}}$ can also be added as columns of the matrix $\boldsymbol{G}$. The resulting code is also balanced but not fully balanced.

B. Example: design of fully balanced STTC with 4-PSK and 3 transmit antennas

For these codes, each generated element belongs to $\mathbb{Z}_{4}^{3}$. It is possible to make the partition of the additive group $\mathbb{Z}_{4}^{3}$ in a normal subgroup $C_{[0]}=2 \mathbb{Z}_{2}^{3}$ of $\mathbb{Z}_{4}^{3}$ and 7 cosets $C_{[p]}=p+C_{[0]}$ with $p \in \mathbb{Z}_{2}^{3} \backslash\left\{\left[\begin{array}{lll}0 & 0 & 0\end{array}\right]^{T}\right\}$.

In this case, there are three types of fully B-STTC [8]:

- Types I: codes with only 1 no null vector in $C_{[0]}$.

- Type II: codes with 2 no null vectors in $C_{[0]}$.

- $\quad$ Type III: codes with 3 no null vectors in $C_{[0]}$.

\section{For type I codes:}

Only the first column $G_{1}=2 p_{1}$ of $\boldsymbol{G}$ belongs to $C_{[0]}$. It generates $H_{1}=\left\{0, C_{1}\right\}$. According to the rule 2, $G_{2}$ must be chosen in the coset $C_{\left[p_{1}\right]}$ relative to $G_{l}$. Thus, $\left\{G_{2}, G_{l}+G_{2}\right\}$ which is a quarter of $C_{\left[p_{1}\right]}$ is also generated. Because no new element of $C_{[0]}$ is generated, the third column must be selected in the same coset $C_{\left[p_{1}\right]}$. After the choice of $G_{3} \in C_{\left[p_{1}\right]} \backslash H_{2}$, half of the coset $C_{\left[p_{1}\right]}$ and half of $C_{[0]}$ are generated. There are two solutions to choose the $4^{\text {th }}$ column:

1) $G_{4} \in C_{\left[p_{1}\right]} \backslash H_{3}$. In this case, the cosets $C_{\left[p_{1}\right]}$ and $C_{[0]}$ are completely generated. The column $G_{5}$ of $\boldsymbol{G}$ is selected in another coset. Due to the choice of $G_{5}$, two new cosets are generated and it remains four cosets to select the last column $G_{6}$.

2) $G_{4}$ is chosen in the cosets relative to the two new generated vectors $G_{2}+G_{3}=2 p_{2}$ and $G_{1}+G_{2}+G_{3}=2 p_{3}$ of $C_{[0]}$. After this choice, half of $C_{[0]}$ and half of three cosets $C_{\left[p_{1}\right]}, C_{\left[p_{2}\right]}$ and $C_{\left[p_{3}\right]}$ are generated. The $5^{\text {th }}$ vector $G_{5}$ are selected in the not generated half of the cosets $C_{\left[p_{2}\right]}$ and $C_{\left[p_{3}\right]}$. After the choice of $G_{5}, C_{[0]}$ and the three cosets are totally generated. The last column $G_{6}$ is chosen in one of the four other cosets.

\section{For type II codes:}

The different no null columns $G_{1}=2 p_{1}$ and $G_{2}=2 p_{2}$ generate $H_{2}=\left\{0, G_{1}, G_{2}, G_{1}+G_{2}\right\}$. The third column $G_{3}$ of $\boldsymbol{G}$ is selected in one of the three cosets $C_{\left[p_{1}\right]}, C_{\left[p_{2}\right]}$ and $C_{\left[p_{1} \oplus p_{2}\right]}$ where $\oplus$ is a sum in $\mathbb{Z}_{2}^{n_{T}}$. After this choice, half of the selected coset and half of $C_{[0]}$ are generated. The choice of the last three columns is similar to the case of type I codes. 
For type III codes:

In this case, 3 no null columns $G_{1}, G_{2}$ and $G_{3}$ selected in $C_{[0]}$ generate entirely $C_{[0]}$. The $4^{\text {th }}$ column of $\boldsymbol{G}$ is selected among one of the other cosets. After this choice, the whole coset is generated. The $5^{\text {th }}$ column of $\boldsymbol{G}$ is selected in one of the 6 other cosets. After this choice, $C_{[0]}$ and three other cosets are generated. It remains four no generated cosets to selecte the last column $G_{6}$ of the coding matrix $\boldsymbol{G}$ of a fully balanced code with $L=L_{\text {min }}$.

\section{Example: design of fully balanced STTC with 8-PSK and} 2 transmit antennas

With this configuration, each MIMO symbol belongs to $\mathbb{Z}_{8}^{2}$ i.e. $Y \in \mathbb{Z}_{8}^{2}$. It is possible to split this group in 16 cosets:

- the coset : $C_{[0]}=4 \mathbb{Z}_{2}^{2}$.

- the set $E_{1}$ of three cosets: $C_{[p]}=p+C_{[0]}$, with $p \in 2 \mathbb{Z}_{2}^{2} \backslash\left\{\left[\begin{array}{ll}0 & 0\end{array}\right]^{T}\right\}$.

- the set $E_{2}$ of twelve cosets $C_{[q]}=q+C_{[0]}$ with $q \in \mathbb{Z}_{4}^{2} \backslash 2 \mathbb{Z}_{2}^{2}$.

To generate a fully balanced code with $\mathrm{L}=\mathrm{L}_{\text {min }}$, i.e. a code with 6 columns, we can proceed as follows :

In agrement with rule 1 , the first no null column $G_{1}=2 p_{1}$ with $p_{1} \in 2 \mathbb{Z}_{2}^{2} \backslash\left\{\left[\begin{array}{ll}0 & 0\end{array}\right]^{T}\right\}$ must be selected in $C_{[0]}$. Thus, the subgroup $H_{1}=\left\{0, C_{1}\right\}$ of $\mathbb{Z}_{8}^{2}$ is generated. As for 4-PSK B-STTC and $n_{T}=2$, there are two types of 8-PSK B-STTC:

- Types I: codes with only 1 no null vector in $C_{[0]}$.

- Types II: codes with 2 no null vectors in $C_{[0]}$.

\section{For type I codes}

The second element must create a new subgroup $H_{2}=\left\{0, G_{1}, G_{2}, G_{1}+G_{2}\right\}$. For this type of code, no other element can be chosen in $C_{[0]}$, therefore $G_{2} \in C_{\left[p_{1}\right]}$. There are two choices to select $G_{3}$ :

- In the same coset of $G_{2}: G_{3} \in C_{\left[p_{1}\right]} \backslash\left\{G_{2}, G_{2}+G_{1}\right\}$. The cosets $C_{[0]}$ and $C_{\left[p_{1}\right]}$ are entirely generated. $G_{4}$ can be chosen :

- either in one of two other cosets of $E_{1}$. In this case, $E_{1}$ is totaly generated. $G_{5}$ is chosen in one of 12 cosets of $E_{2}$. After the choice of $G_{5} \in E_{2}, 4$ cosets of $E_{2}$ are entirely generated and the last column is selected in the 8 nogenerated cosets of $E_{2}$. Thus, a fully balanced code is created.

- either in one of 4 cosets of $E_{2}$ relative to the generated elements of $C_{\left[p_{1}\right]}$. Hence $C_{[0]}, C_{\left[p_{1}\right]}$ and two cosets of $E_{2}$ are generated. $G_{5}$ must be selected in one of the last two no-generated cosets of $E_{2}$ relative to a elements of
$C_{\left[p_{1}\right]}$. It remains 8 no-generated cosets to select the last vector. Hence, a fully balanced code is created.

- In one of two cosets of $E_{2}$ relative to two generated elements of $C_{\left[p_{1}\right]}: G_{1}=2 q_{1}$ and $G_{1}+G_{2}=2 q_{2}$. The half of $C_{[0]}$, of $C_{\left[p_{1}\right]}$ and of two cosets $C_{\left[q_{1}\right]}$ and $C_{\left[q_{2}\right]}$ of $E_{2}$ are generated. $G_{4}$ is selected in the other half of $C_{\left[q_{1}\right]}$ or $C_{\left[q_{2}\right]}$. Due to the choice of $G_{5}, C_{[0]}, C_{\left[p_{1}\right]}, C_{\left[q_{1}\right]}$ and $C_{\left[q_{2}\right]}$ are entirely generated. $G_{5}$ can be selected :

$\circ$ either in $C_{\left[p_{2}\right]} \in E_{1}$ with $C_{\left[p_{2}\right]} \neq C_{\left[p_{1}\right]} . C_{[0]}, E_{1}$ and 4 cosets of $E_{2}$ are entirely generated. The last vector is chosen in one of 8 no-generated cosets of $E_{2}$.

- either in one of the cosets of $E_{2}$ relative to the two new generated elements of $C_{\left[p_{1}\right]}$. Thus $C_{[0]}, E_{l}$ and 4 cosets of $E_{2}$ are entirely generated. The last column is chosen in one of 8 no-generated cosets of $E_{2}$.

\section{For type II codes}

The second column of $\boldsymbol{G}$ must be selected in $C_{[0]}$. These two selected elements generate $C_{[0]}$. The third column is selected in one of cosets of $E_{1}$. Hence, $C_{[0]}$ and this coset are totaly generated. There are two choices to select $G_{4}$ :

- $\quad$ either in one of two other cosets of $E_{1} . C_{[0]}$ and the set $E_{1}$ are totaly generated. $G_{5}$ is chosen in $E_{2}$. Thus $C_{[0]}, E_{1}$ and 4 cosets of $E_{2}$ are generated. The last column of $\boldsymbol{G}$ is chosen in one of the no-generated cosets of $E_{2}$. Hence, a balanced code of Type II is created.

- $\quad$ either in one of 4 cosets relative to the generated elements of $E_{1}$. Then $C_{[0]}$, one coset of $E_{1}$ and 2 cosets of $E_{2}$ are generated. It remains 2 no-generates cosets relative to the generated elements of $E_{1} . G_{5}$ must be selected in these cosets. $C_{[0]}, E_{1}$ and 4 cosets of $E_{2}$ are entirely generated.

The last column is chosen in one of the 8 no-generated cosets of $E_{2}$.

\section{CODE PERFormance}

After the systematic search in the class of balanced codes, new codes with good performance have been found for 3 and 4 transmit antennas and several number of states. A new 16 states 4-PSK STTC is reported in Table I obtained with rank and determinant criteria.

Table II presents new codes with larger trace than the Chen's code [10]. The codes noted by "FB" are fully balanced, those noted by "B" are not balanced and those noted by "NB" are not balanced. Each new code is balanced and its trace is higher than the trace of Chen's corresponding code. 
TABLE I. CODES BASED ON THE RDC

\begin{tabular}{|c|c|c|c|}
\hline $\mathrm{n}_{\mathrm{T}}$ & States & Chen $[10]$ & New 1 \\
\hline \multirow{3}{*}{3} & \multirow{3}{*}{16} & {$\left[\begin{array}{llllll}0 & 2 & 0 & 1 & 2 & 2 \\
0 & 0 & 1 & 2 & 3 & 3 \\
2 & 0 & 2 & 0 & 1 & 3\end{array}\right]$} & {$\left[\begin{array}{llllll}0 & 1 & 2 & 2 & 2 & 0 \\
0 & 2 & 0 & 1 & 2 & 2 \\
2 & 2 & 0 & 2 & 3 & 0\end{array}\right]$} \\
& \multirow{3}{*}{ NB } & FB \\
\hline \multicolumn{2}{|c|}{3} & $32 / 246.5$ & $32 / 198.2$ \\
\hline Rank min & 32 & 3 \\
\hline
\end{tabular}

TABLE II. CODES BASED ON THE EDC

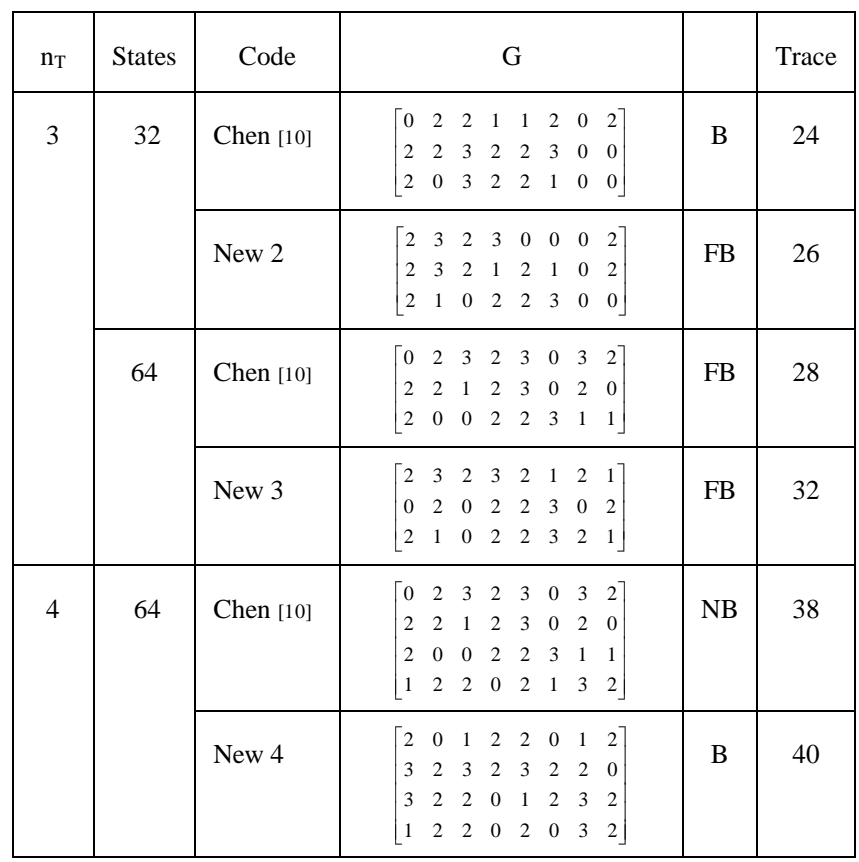

The performance of each code is evaluated by simulation in a slow Rayleigh fading channel. The fading coefficients are independant samples of a complex Gaussian process with mean zero and variance 0.5 per dimension. Each frame consists of 130 4-PSK symbols. Fig. 1 shows the performance of the 4-PSK 16-state codes with 3 transmit antennas of table I. The new fully balanced code outperform the Chen's code in the case of one receive antenna.

Fig 2. shows the performance of Chen's codes and new codes based on the euclidian distance criterion for 3 transmit antennas and 2 receive antennas, for 32/64 states. As previously, the new balanced STTC codes outperform the corresponding best known codes proposed by Chen.

\section{CONCLUSION}

It has been noticed that the good space-time trellis codes belong to the class of balanced STTC. These codes generate the points of the MIMO constellation with the same probability. In this paper, a general and simple method to design 4-PSK and 8-PSK STTC with $n_{T}$ transmit antennas and several numbers of states has been proposed. The systematic search of good STTC can be reduced to this class. Finally, some new balanced 16/32/64 states codes which outperform the best previously published codes have also been proposed.

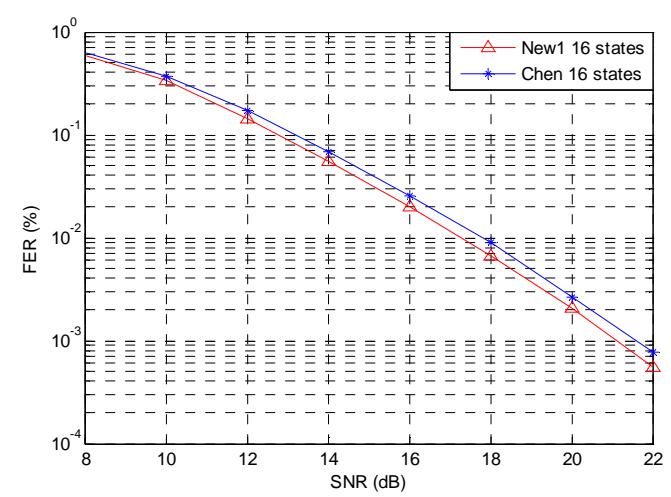

Fig. 1. Performance of 4-PSK STTC with $3 \mathrm{Tx} / 1 \mathrm{Rx}$ antennas.

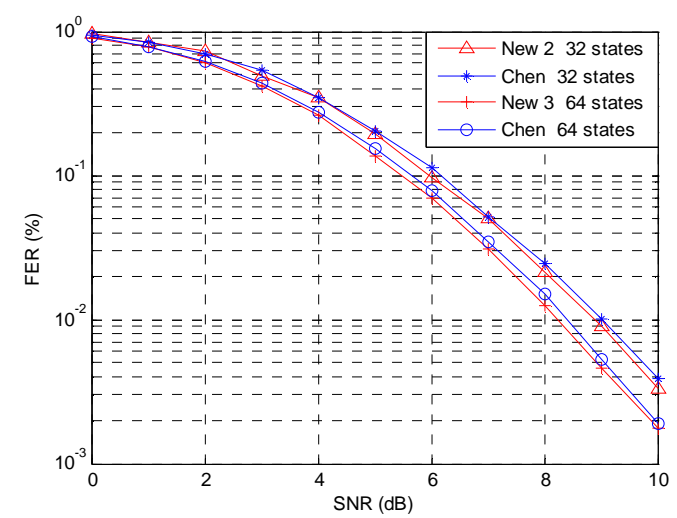

Fig. 2. Performance of 4-PSK STTC with $3 \mathrm{Tx} / 2 \mathrm{Rx}$ antennas.

\section{REFERENCES}

[1] V. Tarokh, N. Seshadri, and A.R. Calderbank, "Space-time codes for high data rate wireless communication: Performance criterion and code construction", IEEE Trans. Inform. Theory, vol. 44, no. 2, pp. 744-765, March 1998.

[2] Z. Chen, J. Yuan, and B. Vucetic, "Improved space-time trellis coded modulation scheme on slow fading channels", Electron. Lett., vol. 37, no. 7, pp. 440-441, March 2001.

[3] W. Firmanto, B.S. Vucetic, and J. Yuan, "Space-time TCM with improved performance on fast fading channels", IEEE Commun. Lett., vol. 5, no. 4, pp. 154-156, April 2001.

[4] S. Baro, G. Bauch, and A. Hansmann, "Improved codes for space-time trellis-coded modulation", IEEE Commun. Lett., vol. 4, no. 1, pp. 20-22, Jan. 2000.

[5] Yi Hong and A. Guillen i Fàbregas, "New Space-Time Trellis Codes for Slow Fading Channels", in Proc. IEEE VTC 2006-Spring, vol. 3, pp. 1492-1496, May 2006.

[6] Y.S. Jung and J.H. Lee, "Improved space-time trellis codes using expanded signal set in slow Rayleigh fading channels", in Proc. IEEE ISIT'2002, pp. 248, June 2002.

[7] Z. Chen, B.S. Vucetic, J. Yuan, and Ka Leong Lo, "Space-time trellis codes for 4-PSK with three and four transmit antennas in quasi-static flat fading channels," IEEE Commun. Lett., vol. 6, no. 2, pp. 67-69, Feb. 2002.

[8] T.M.H. Ngo, G. Zaharia, S. Bougeard and J.F. Helard, "A new class of balanced 4-PSK STTC for two and three transmit antennas ", $8^{\text {th }}$ Workshop on SPAWC 2007, pp. 1-5, June 2007.

[9] Chen Liao, V.K. Prabhu "Improved code design criteria for space-time trellis codes overs quasi-static flat fading channels", $6^{\text {th }}$ Workshop on SPAWC 2005, pp. 7-11, June 2005.

[10] Z. Chen, B. Vucetic, J. Yuan and K.L. Lo, "Space-time trellis codes with two, three and four transmit antennas in quasi-static flat fading channels", in Proc. ICC'2002, vol. 3, pp. 1589-1595, May 2002. 